M. Dehghani, Z. Montazeri, O.P. Malik

\title{
ENERGY COMMITMENT: A PLANNING OF ENERGY CARRIER BASED ON ENERGY CONSUMPTION
}

\begin{abstract}
Purpose. Energy consumption is one of the criteria for determining the quality of life in a country. Continued supply of energy and the possibility of long-term access to resources require a comprehensive plan. One of the key issues in the field of energy planning is energy carriers. In this paper, a new theory is introduced to energy network studies for planning of energy carriers called Energy Commitment. In this theory, an appropriate planning is applied for energy carriers based the final energy consumption. Energy carriers are available either naturally or after the energy conversion process. Energy commitment is modeled on an energy network with the presence of electrical energy, gas energy, transportation section, agriculture section, industrial section, residential section, commercial section, and general section. References 25, tables 3 . Key words: energy, energy commitment, energy carrier, energy consumption, unit commitment.
\end{abstract}

Цель. Потребление энергии является одним из критериев определения качества жкизни в стране. Непрерывные поставки энергии и возможсность долгосрочного доступа к ресурсам требуют комплексного плана. Одним из ключевых вопросов в области энергетического планирования являются энергоносители. В данной статье в исследования энергетических сетей для планирования энергоносителей вводится новая теория под названием Епеrgy Соттіттепt («энергетическое обязательство»). В этой теории для энергоносителей применяется соответствующее планирование на основе конечного потребления энергии. Энергоносители доступны либо естественным путем, либо после процесса преобразования энергии. Energy Commitment моделируется в энергетической сети с учетом электрической энергии, энергии газа, транспортной отрасли народного хозяйства, сельскохозяйственной отрасли, промышленного сектора экономики, жсилищнокоммунального хозяйства, реального сектора экономики и прочих видов экономической активности. Библ. 25 , табл. 3. Ключевые слова: энергия, энергетическое обязательство, энергоноситель, энергопотребление, единичное обязательство.

Introduction. Energy consumption is one of the criteria for determining the level of development and quality of life in a country [1]. If energy used properly and reasonably, it can in any country make progress in the science, technology and welfare of its people. Otherwise, it will cause irreparable economic losses and a massive economic downturn [2]. The energy consumption trend has been very fast and critical in recent years. Continued supply of energy and the possibility of long-term access to resources require a comprehensive energy planning, which is why energy planning is indisputable economic, national and strategic imperatives. One of the key issues in the field of energy planning is energy resources.

Many studies is done on the power system such as: transformers [3], battery energy storage [4], distributed generation [5], energy [6]. One of the most important studies of electric power network is the issue of Unit Commitment (UC) [7]. UC is to determine the most appropriate electrical power generation pattern at power plants, firstly, to meet technical requirements, and then to be the most economical [8]. UC has been studied using various methods. The priority list method and dynamic programing are the first methods in UC [9]. In the Lagrange method, equal and unequal constraints were added to the objective function [10]. In [11] UC problem is investigated the in presence of FACTS devices and energy storage. In [12] UC problem is studied under cyber-attacks. In addition, evolutionary methods have been used for solving UC in recent years. In [13] a method is proposed based on the classical genetic algorithm. Integer-coded genetic algorithm in [14] is proposed. Researchers have also used other methods to solve the UC problem such as: Particle Swarm Optimization (PSO) [15], Teaching Learning Based Optimization (TLBO) [16], Gravitational Search Algorithm (GSA) [17], Water Cycle Algorithm (WCA) [18] and Grey Wolf Optimization (GWO) [19], Whale
Optimization Algorithm (WOA) [20]. Other algorithms are also suggested for UC solving [21-24].

Energy Commitment (EC) is to determine the most appropriate pattern for using energy resources to meet energy demand, firstly, to meet technical requirements, and secondly, to be the most economical. In other words, energy sources should be used as much as needed, if the energy sources are in line with the demand peak it will cost a lot. Therefore, EC reduces energy supply costs.

This problem can be articulated mathematically, so that a function called $F$ is defined as the objective function, which is equal to the total cost of supplying energy demand. In this case, the problem is to minimize $F$. Note that losses are discarded and there is no explicit mention of any exploitation restrictions in the issue. So:

$$
\begin{aligned}
& F=F_{1}\left(E_{s_{1}}\right)+F_{2}\left(E_{s_{2}}\right)+F_{3}\left(E_{s_{13}}\right)+ \\
& +\ldots+F_{N_{s}}\left(E_{S_{N_{s}}}\right)=\sum_{i=1}^{N_{s}} F_{i}\left(E_{s_{i}}\right),
\end{aligned}
$$

where $F$ is the objective function, $F_{i}$ is the cost of $i$-th source, $E_{s_{i}}$ is the $i$-th kind of energy demand and $N_{s}$ is the number of energy carriers.

The above issue is an optimization problem that can be examined using appropriate methods.

Problem Formulation. Energy grid modelling. The energy network consists of the following sections: transportation, agriculture, industrial, residential, commercial and general.

In the energy grid, energy demand is calculated as a sum of sub networks of the grid:

$$
E C_{f}=E C_{1}+E C_{2}+\ldots+E C_{N}=\sum_{i=1}^{N} E C_{i},
$$

where $E C_{f}$ is the final energy consumption, $N$ is the number of different sections of energy consumption and $E C_{i}$ is the energy consumption of $i$-th section. 
Firstly, the final energy consumption matrix based on different sections is determined as

$$
E_{1}=\left[\begin{array}{llllll}
E C_{1} & E C_{2} & \ldots & E C_{i} & \ldots & E C_{N}
\end{array}\right]^{T},
$$

where $E_{1}$ is the final energy consumption matrix based on different sections.

Now final energy consumption matrix based on different energy carriers is determined as

$$
E_{2}=T_{1,2} \times E_{1} \text {, }
$$

where $E_{2}$ is the final energy consumption matrix based on different energy carriers and $T_{1,2}$ is the transpose matrix of different sections to different energy carriers.

Energy losses is modeled as

$$
E_{3}=T_{2,3} \times E_{2} \text {, }
$$

where $E_{3}$ is the final energy consumption based on different energy carriers considering losses and $T_{2,3}$ is the efficiency matrix.

At this stage, electrical energy is converted into energy carriers. The electrical energy of different power plants is determined as

$$
E_{u}=T_{u} \times E_{e},
$$

where $E_{u}$ is the electrical energy of different power plants, $T_{u}$ is the separation matrix of electricity generation by different power plants and $E_{e}$ is the total electricity demand.

Input fuel for different power plants is determined as

$$
E_{e_{1}}=T_{u, f} \times E_{u},
$$

where $E_{e_{1}}$ is the input fuel for different power plant and Electrical manufacturer carriers is determined as

$$
E_{e_{2}}=T_{f, c} \times E_{e_{1}},
$$

where $E_{e_{2}}$ is the electrical manufacturer carriers and $T_{f, c}$ is the conversion matrix of input fuel to energy carriers.
After simulation of electrical energy, final energy consumption is calculated as

$$
E_{4}=E_{3}+E_{e_{2}}-E_{e}
$$

where $E_{4}$ is the final energy consumption after conversion of electrical energy.

At this stage, the process of refining crude oil is simulated as

$$
E_{p_{1}}=T_{p} \times E_{p}
$$

where $E_{p_{1}}$ is the energy carriers produced by refining, $T_{p}$ is the separation matrix of produced products from refining crude oil and $E_{p}$ is the maximum capacity of refineries.

After simulation of process of refining crude oil, final energy consumption is calculated as

$$
E_{5}=E_{4}+E_{p}-E_{p_{1}},
$$

where $E_{5}$ is the final energy consumption after refining crude oil. Actually $E_{5}$ determines energy carriers in order to supply of energy demand.

Test energy grid. EC is applied to energy grid with 10 power units. Electrical network information is adapted from [25].

Simulation. After modeling the energy network, EC is simulated on energy grid.

The simulation results of EC on the energy grid studied are presented in Tables 1-3.

In Table 1, dynamic scheduling results are presented

\begin{tabular}{|c|c|c|c|c|c|c|}
\hline \multicolumn{6}{|c|}{ Strategy } & \multirow{2}{*}{ Hour } \\
\hline S6 & S5 & S 4 & S3 & S2 & $\mathrm{S} 1$ & \\
\hline 2 & 2 & 2 & 2 & 2 & 2 & The initial state \\
\hline 3 & 3 & 3 & 3 & 3 & 3 & 1 \\
\hline 3 & 3 & 3 & 3 & 3 & 3 & 2 \\
\hline 3 & 3 & 3 & 3 & 3 & 3 & 3 \\
\hline 3 & 3 & 3 & 3 & 3 & 3 & 4 \\
\hline 3 & 3 & 3 & 3 & 3 & 3 & 5 \\
\hline 4 & 4 & 4 & 4 & 4 & 4 & 6 \\
\hline 4 & 4 & 4 & 4 & 4 & 4 & 7 \\
\hline 9 & 9 & 9 & 9 & 9 & 9 & 8 \\
\hline 9 & 9 & 9 & 9 & 9 & 9 & 9 \\
\hline 9 & 9 & 9 & 9 & 9 & 9 & 10 \\
\hline 10 & 10 & 10 & 10 & 10 & 10 & 11 \\
\hline 10 & 10 & 10 & 10 & 10 & 10 & 12 \\
\hline 10 & 10 & 10 & 10 & 10 & 10 & 13 \\
\hline 9 & 9 & 9 & 9 & 9 & 9 & 14 \\
\hline 9 & 9 & 9 & 9 & 9 & 9 & 15 \\
\hline 9 & 9 & 9 & 9 & 9 & 9 & 16 \\
\hline 9 & 9 & 9 & 9 & 9 & 9 & 17 \\
\hline 9 & 9 & 9 & 9 & 9 & 9 & 18 \\
\hline 9 & 9 & 9 & 9 & 9 & 9 & 19 \\
\hline 9 & 9 & 9 & 9 & 9 & 9 & 20 \\
\hline 9 & 9 & 4 & 4 & 4 & 4 & 21 \\
\hline 9 & 6 & 4 & 4 & 3 & 3 & 22 \\
\hline 7 & 6 & 4 & 4 & 3 & 3 & 23 \\
\hline 7 & 6 & 5 & 4 & 3 & 2 & 24 \\
\hline $8,557,932$ & $8,557,192$ & $8,557,153$ & $8,554,502$ & $8,554,182$ & $8,555,398$ & Cost (USD) \\
\hline
\end{tabular}
with equal paths to the maximum number of states per hour of the study. The second path, (S2) is identified as an appropriate strategy. The cost of EC in this path is equal by $8,554,182$ USD. The need for energy carriers to provide final energy consumption is specified in Table 2 . The result of economic distribution of electrical energy is presented in Table 3.

The output result of dynamic planning in ten unit energy grids

Table 1 
Table 2

The need of energy carriers in ten unit energy grids

\begin{tabular}{|c|c|c|c|c|c|c|c|c|}
\hline 8 & 7 & 6 & 5 & 4 & 3 & 2 & 1 & Hour \\
\hline 3721.1 & 3721.1 & 3721.1 & 3721.1 & 3721.1 & 3721.1 & 3721.1 & 3721.1 & Petroleum \\
\hline 51.78965 & 4.67028 & 37.55091 & 3.31218 & 16.19281 & 1.95407 & -12.2847 & -19.404 & Liquid gas \\
\hline-350.552 & -365.265 & 354.657 & -429.906 & -466.355 & -539.254 & -612.154 & -647.68 & \\
\hline-11.7441 & -61.1345 & 123.351 & -210.1 & -253.46 & -340.182 & -426.903 & -470.252 & Gas oil \\
\hline 17.72885 & 1.640607 & -14.4476 & -46.6241 & -62.7124 & -94.8888 & -127.065 & -143.154 & ene \\
\hline 405.1893 & 363.9642 & 22.7392 & 240.289 & 199.0639 & 116.6137 & 34.16357 & -7.06152 & line \\
\hline 53.06305 & 50.85209 & 8.64113 & 44.2192 & 42.00824 & 37.58632 & 33.1644 & 30.95344 & fuel \\
\hline 4380.603 & 4190.728 & 3988.239 & 3615.204 & 3432.123 & 3065.959 & 2699.796 & 2519.415 & Natural gas \\
\hline 26.60254 & 25.4941 & 4.38566 & 22.16878 & 21.06034 & 346 & 16.62658 & 15.51815 & gas \\
\hline 58.79772 & 56.34781 & 53.89791 & 48.9981 & 46.54819 & 41.64838 & 36.74857 & 34.29867 & Coal \\
\hline 16 & 15 & 14 & 13 & 12 & 11 & 10 & 9 & Hour \\
\hline 3721.1 & 721.1 & 3721.1 & 3721.1 & 3721.1 & 3721.1 & 3721.1 & 3721.1 & Petroleum \\
\hline 30.43155 & .78965 & 66.02839 & 80.26713 & 94.50586 & 87.3865 & 80.26713 & 66.02839 & Liquid gas \\
\hline-459.901 & -350.552 & -275.868 & -198.861 & -135.511 & -158.969 & -198.861 & -275.591 & Fuel oil \\
\hline-141.826 & -11.7441 & 74.99814 & 161.7678 & 260.843 & 205.169 & 161.7678 & 75.0014 & Gas oil \\
\hline-30.5359 & 17.72885 & 49.90533 & 82.0818 & 114.2583 & 98.17004 & 82.0818 & 49.90533 & Kerosene \\
\hline 281.5141 & 405.1893 & 487.6395 & 570.0897 & 652.5398 & 611.3148 & 570.0897 & 487.6395 & Gasoline \\
\hline 46. & 53.06305 & & & & & & 3497 & fuel \\
\hline 3831.358 & 4380.603 & 4751.988 & 5130.168 & 5531 & 5323.32 & 5130.168 & 4752.798 & Natural gas \\
\hline 23.27722 & 26.60254 & 28.81941 & 31.03629 & 33.25317 & 32.14473 & 31.03629 & 28.81941 & Coke gas \\
\hline 51.448 & 8.79772 & 63.69753 & 68.59734 & 73.49714 & 71.04724 & 68.59734 & 63.69753 & Coal \\
\hline 24 & 23 & 22 & 21 & 20 & 19 & 18 & 17 & Hour \\
\hline 3721.1 & 721.1 & 3721.1 & 721.1 & 3721.1 & 3721.1 & 3721.1 & 3721.1 & leum \\
\hline-5.1653 & 9.073439 & 37.55091 & 66.02839 & 80.26713 & 51.78965 & 37.55091 & 23.31218 & Liquid gas \\
\hline-595.486 & -548.095 & -423.452 & -275.868 & -198.861 & -350.552 & -423.452 & -496.351 & Fuel oil \\
\hline-370.456 & -277.548 & -98.4652 & 74.99813 & 161.7678 & -11.7441 & -98.4652 & -185.186 & Gas oil \\
\hline-110.977 & -78.8006 & -14.4476 & 49.90533 & 82.0818 & 17.72885 & -14.4476 & -46.6241 & Kerosene \\
\hline 75.38865 & 157.8388 & 322.7392 & 487.6395 & 570.0897 & 405.1893 & 322.7392 & 240.289 & Gasoline \\
\hline 35.37536 & 39.79728 & 48.64113 & 57.48497 & 61.90689 & 53.06305 & 48.64113 & 44.2192 & Plane fuel \\
\hline 2913.867 & 3278.051 & 4014.44 & 4751.988 & 5130.168 & 4380.603 & 4014.44 & 3648.277 & Natural gas \\
\hline 17.73502 & 19.9519 & 24.38566 & 28.81941 & 31.03629 & 26.60254 & 24.38566 & 22.16878 & Coke gas \\
\hline 39.19848 & 44.09829 & 53.89791 & 63.69753 & 68.59734 & 58.79772 & 53.89791 & 48.9981 & Coal \\
\hline
\end{tabular}

Table 3

The electrical energy economical distribution within the energy grid

\begin{tabular}{|c|c|c|c|c|c|c|c|c|c|c|}
\hline $\begin{array}{l}\stackrel{0}{\varrho} \\
. \\
.\end{array}$ & 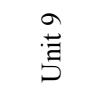 & $\stackrel{\infty}{\stackrel{\infty}{\mid}}$ & $\stackrel{\sim}{.}$ & $\stackrel{\bullet}{\stackrel{*}{3}}$ & $\stackrel{n}{\stackrel{B}{G}}$ & 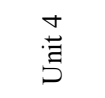 & 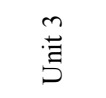 & 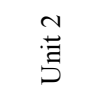 & 壱 & $\stackrel{\Xi}{\Xi}$ \\
\hline 0 & 0 & 0 & 0 & 0 & 0 & 0 & 129.9054 & 150 & 420.9897 & 1 \\
\hline 0 & 0 & 0 & 0 & 0 & 0 & 0 & 130 & 165.9591 & 455 & 2 \\
\hline 0 & 0 & 0 & 0 & 0 & 0 & 0 & 130 & 266.087 & 455 & 3 \\
\hline 0 & 0 & 0 & 0 & 0 & 0 & 0 & 130 & 366.2149 & 455 & 4 \\
\hline 0 & 0 & 0 & 0 & 0 & 0 & 0 & 130 & 416.2788 & 455 & 5 \\
\hline 0 & 0 & 0 & 0 & 0 & 0 & 61.40668 & 130 & 455 & 455 & 6 \\
\hline 0 & 0 & 0 & 0 & 0 & 0 & 111.4706 & 130 & 455 & 455 & 7 \\
\hline 0 & 54.94904 & 10 & 25 & 78.91501 & 25 & 20 & 129.9395 & 403.1555 & 454.5755 & 8 \\
\hline 0 & 54.92522 & 38.19602 & 25 & 79.91727 & 25 & 40.51524 & 129.8847 & 454.393 & 453.831 & 9 \\
\hline 0 & 54.99011 & 46.54565 & 75.69185 & 79.97855 & 25 & 129.9675 & 129.966 & 454.8779 & 454.8368 & 10 \\
\hline 55 & 55 & 55 & 85 & 80 & 51.98213 & 130 & 130 & 455 & 455 & 11 \\
\hline 55 & 55 & 55 & 85 & 80 & 157.1164 & 130 & 130 & 455 & 455 & 12 \\
\hline 31.11385 & 55 & 55 & 85 & 80 & 25.80435 & 130 & 130 & 455 & 455 & 13 \\
\hline 0 & 55 & 46.5999 & 25.09276 & 80 & 25.18803 & 130 & 130 & 455 & 454.9096 & 14 \\
\hline 0 & 50.46745 & 10 & 25 & 42.35772 & 25 & 20 & 129.0834 & 452.7482 & 446.8778 & 15 \\
\hline 0 & 54.57776 & 10 & 25 & 75.61226 & 25 & 20 & 129.572 & 260.4829 & 451.0978 & 16 \\
\hline 0 & 54.58248 & 10 & 25 & 75.74856 & 25 & 20 & 129.4813 & 209.902 & 451.5645 & 17 \\
\hline 0 & 55 & 10.06585 & 25.04071 & 80 & 25.08315 & 20.12963 & 130 & 401.2152 & 455 & 18 \\
\hline 0 & 55 & 46.61355 & 25.03679 & 80 & 25.13997 & 130 & 130 & 455 & 455 & 19 \\
\hline 0 & 53.36535 & 10 & 25 & 79.89353 & 25 & 70.70835 & 129.7906 & 454.3342 & 453.5704 & 20 \\
\hline 0 & 0 & 0 & 0 & 0 & 0 & 61.40668 & 130 & 455 & 455 & 21 \\
\hline 0 & 0 & 0 & 0 & 0 & 0 & 0 & 130 & 316.1509 & 455 & 22 \\
\hline 0 & 0 & 0 & 0 & 0 & 0 & 0 & 130 & 216.023 & 455 & 23 \\
\hline 0 & 0 & 0 & 0 & 0 & 0 & 0 & 130 & 216.023 & 455 & 24 \\
\hline
\end{tabular}

\section{Conclusions.}

Energy Commitment (EC) was introduced as a planning of energy carrier based on energy consumption. $\mathrm{EC}$ is to determine the most appropriate pattern for using energy resources to meet energy demand, firstly, to meet technical requirements, and secondly, to be the most economical.

The energy grid including different sections was modeled in matrix form. EC was simulated on the one energy grid with ten power plants and result was 
presented. Different combinations of power plants are available to provide final energy consumption. Due to the different fuel inputs to each power plant, there are different combinations of energy carriers. The proper combination of energy carriers is determined to provide final energy consumption using the dynamic programming method.

\section{REFERENCES}

1. Dehghani M., Montazeri Z., Ehsanifar A., Seifi A.R., Ebad M.J., Grechko O.M. Planning of energy carriers based on final energy consumption using dynamic programming and particle swarm optimization. Electrical engineering \& electromechanics, 2018, no.5, pp. 62-71. doi: 10.20998/2074-272X.2018.5.10.

2. Montazeri Z., Niknam T. Energy carriers management based on energy consumption. 2017 IEEE 4th International Conference on Knowledge-Based Engineering and Innovation (KBEI), Dec. 2017. doi: 10.1109/kbei.2017.8325036.

3. Ehsanifar A., Dehghani M., Allahbakhshi M. Calculating the leakage inductance for transformer inter-turn fault detection using finite element method. 2017 Iranian Conference on Electrical Engineering (ICEE), May 2017. doi: 10.1109/iraniancee.2017.7985256.

4. Dehbozorgi S., Ehsanifar A., Montazeri Z., Dehghani M., Seifi A. Line loss reduction and voltage profile improvement in radial distribution networks using battery energy storage system. 2017 IEEE 4th International Conference on Knowledge-Based Engineering and Innovation (KBEI), Dec. 2017. doi: 10.1109/kbei.2017.8324976.

5. Dehghani M., Mardaneh M., Montazeri Z., Ehsanifar A., Ebadi M.J., Grechko O.M. Spring search algorithm for simultaneous placement of distributed generation and capacitors. Electrical engineering \& electromechanics, 2018, no.6, pp. 6873. doi: 10.20998/2074-272X.2018.6.10.

6. Montazeri Z., Niknam T. Optimal utilization of electrical energy from power plants based on final energy consumption using gravitational search algorithm. Electrical engineering \& electromechanics, 2018, no.4, pp. 70-73. doi: 10.20998/2074272X.2018.4.12.

7. Shi J., Oren S.S. Stochastic Unit Commitment With Topology Control Recourse for Power Systems With Large-Scale Renewable Integration. IEEE Transactions on Power Systems, 2018, vol.33, no.3, pp. 3315-3324. doi: 10.1109/tpwrs.2017.2772168.

8. Gupta A., Anderson C.L. Statistical Bus Ranking for Flexible Robust Unit Commitment. IEEE Transactions on Power Systems, 2019, vol.34, no.1, pp. 236-245. doi: 10.1109/tpwrs.2018.2864131. 9. Yamin H.Y. Review on methods of generation scheduling in electric power systems. Electric Power Systems Research, 2004 vol.69, no.2-3, pp. 227-248. doi: 10.1016/j.epsr.2003.10.002.

10. Geoffrion A.M. Lagrangian Relaxation for Integer Programming. 50 Years of Integer Programming 1958-2008. Nov. 2009, pp. 243-281, doi:10.1007/978-3-540-68279-0_9.

11. Luburić Z., Pandžić H. FACTS devices and energy storage in unit commitment. International Journal of Electrical Power \& Energy Systems, 2019, vol.104, pp. 311-325 doi: 10.1016/j.ijepes.2018.07.013.

12. Shayan H., Amraee T. Network Constrained Unit Commitment Under Cyber Attacks Driven Overloads. IEEE Transactions on Smart Grid, pp. 1-1, 2019. doi: 10.1109/tsg.2019.2904873.

13. Swarup K.S., Yamashiro S. Unit commitment solution methodology using genetic algorithm. IEEE Transactions on Power Systems, 2002, vol.17, no.1, pp. 87-91. doi: 10.1109/59.982197.

14. Damousis I.G., Bakirtzis A.G., Dokopoulos P.S. A Solution to the Unit-Commitment Problem Using Integer-Coded Genetic Algorithm. IEEE Transactions on Power Systems, 2004, vol.19, no.2, pp. 1165-1172. doi: 10.1109/tpwrs.2003.821625.
15. Anand H., Narang N., Dhillon J.S. Multi-objective combined heat and power unit commitment using particle swarm optimization. Energy, 2019, vol.172, pp. 794-807. doi: 10.1016/j.energy.2019.01.155.

16. Krishna P.V.R., Sao S. An Improved TLBO Algorithm to Solve Profit Based Unit Commitment Problem under Deregulated Environment. Procedia Technology, 2016, vol.25, pp. 652-659. doi: 10.1016/j.protcy.2016.08.157.

17. Barani F., Mirhosseini M., Nezamabadi-pour H., Farsangi M.M. Unit commitment by an improved binary quantum GSA. Applied Soft Computing, 2017, vol.60, pp. 180-189. doi: 10.1016/j.asoc.2017.06.051.

18. El-Azab H.-A.I., Swief R.A.-W., El-Amary N.H., Temraz H.K. Decarbonized Unit Commitment Applying Water Cycle Algorithm Integrating Plug-In Electric Vehicles. 2018 Twentieth International Middle East Power Systems Conference (MEPCON), Dec. 2018. pp. 455-462. doi: 10.1109/mepcon.2018.8635152.

19. Srikanth K., Panwar L.K., Panigrahi B., Herrera-Viedma E., Sangaiah A.K., Wang G.-G. Meta-heuristic framework: Quantum inspired binary grey wolf optimizer for unit commitment problem. Computers \& Electrical Engineering, 2018, vol.70, pp. 243-260. doi: 10.1016/j.compeleceng.2017.07.023.

20. Kumar V., Kumar D. Binary whale optimization algorithm and its application to unit commitment problem. Neural Computing and Applications, Oct. 2018, pp. 1-29, doi: 10.1007/s00521-018-3796-3. 21. Dehghani M., Montazeri Z., Dehghani A., Nouri N., Seifi A. BSSA: Binary spring search algorithm. 2017 IEEE 4th International Conference on Knowledge-Based Engineering and Innovation (KBEI), Dec. 2017. doi: 10.1109/kbei.2017.8324977.

22. Dehghani M., Montazeri Z., Dehghani A., Seifi A. Spring search algorithm: A new meta-heuristic optimization algorithm inspired by Hooke's law. 2017 IEEE 4th International Conference on Knowledge-Based Engineering and Innovation (KBEI), Dec. 2017. doi: 10.1109/kbei.2017.8324975.

23. Dehghani M., Montazeri Z., Malik O.P., Ehsanifar A., Dehghani A. OSA: Orientation Search Algorithm. International Journal of Industrial Electronics, Control and Optimization, 2019, vol.2, pp. 99-112.

24. Dehghani M., Mardaneh M., Malik O. FOA: Following Optimization Algorithm for solving power engineering optimization problems. Journal of Operation and Automation in Power Engineering, 2019. (Article in press). doi: 10.22098/JOAPE.2019.5522.1414.

25. Ebrahimi J., Hosseinian S.H., Gharehpetian G.B. Unit Commitment Problem Solution Using Shuffled Frog Leaping Algorithm. IEEE Transactions on Power Systems, 2011, vol.26, no.2, pp. 573-581. doi: 10.1109/tpwrs.2010.2052639.

Received 19.04.2019

M. Dehghani ${ }^{1}$, Candidate of Power Engineering, PhD Student, Z. Montazeri ${ }^{1}$, Candidate of Power Engineering, PhD Student, O.P. Malik2, Doctor of Power Engineering, Professor,

${ }^{1}$ Department of Electrical and Electronics Engineering,

Shiraz University of Technology, Shiraz, Iran,

e-mail: adanbax@gmail.com, Z.Montazeri@sutech.ac.ir

${ }^{2}$ Department of Electrical Engineering,

University of Calgary, Calgary Alberta Canada

e-mail: maliko@ucalgary.ca

\section{How to cite this article:}

Dehghani M., Montazeri Z., Malik O.P. Energy commitment: a planning of energy carrier based on energy consumption. Electrical engineering \& electromechanics, 2019, no.4, pp. 69-72. doi: 10.20998/2074-272X.2019.4.10. 